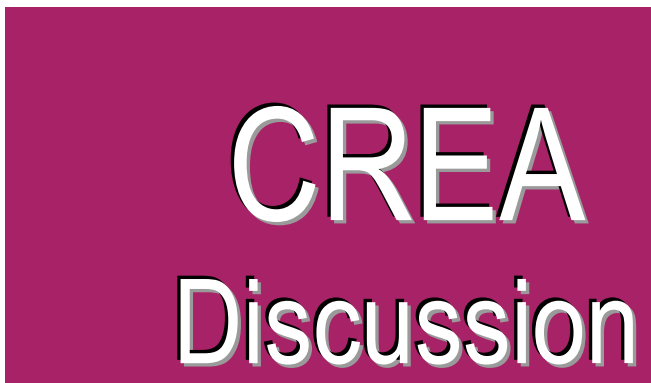
Paper 2018-22 Economics

\title{
Tasks, Technology, and Factor Prices in the Neoclassical Production Sector
}

available online : http://wwwfr.uni.lu/recherche/fdef/crea/publications2/discussion_papers

Andreas Irmen, CREA, Université du Luxembourg

December, 2018

For editorial correspondence, please contact: crea@uni.lu

University of Luxembourg

Faculty of Law, Economics and Finance

$162 \mathrm{~A}$, avenue de la Faïencerie L-1511 Luxembourg 


\title{
TASKS, TECHNOLOGY, AND FACTOR PRICES \\ IN THE \\ NEOCLASSICAL PRODUCTION SECTOR
}

\author{
Andreas Irmen* \\ University of Luxembourg and CESifo, Munich
}

\begin{abstract}
This paper introduces tasks into the neoclassical production sector. Competitive firms choose the profit-maximizing amounts of factor-specific tasks that determine their factor demands and output supplies. We show that the effect of factor-augmenting technical change on relative and absolute factor prices can be decomposed into a productivity effect and a market size effect of opposite sign. These effects appear since the novel task-based approach distinguishes between the demands for tasks and the demands for factors. This perspective provides a new intuition for the emergence of relative and absolute factor biases and the role of the elasticity of substitution.
\end{abstract}

Keywords: Technical Change, Factor Prices, Factor-Specific Tasks, Neoclassical Production.

JEL-Classification: $\mathrm{O}_{33}, \mathrm{O}_{41}$.

This Version: December 19, 2018.

\footnotetext{
${ }^{*}$ CREA, University of Luxembourg, Faculty of Law, Economics and Finance, 162a, avenue de la Faïencerie, L-1511 Luxembourg, Luxembourg, airmen@uni.lu.

I gratefully acknowledge financial assistance under the Inter Mobility Program of the FNR Luxembourg ("Competitive Growth Theory - CGT"). A part of this research was written while I was visiting Brown University in the Spring 2018. I would like to express my gratitude to Brown's Economics Department for its kind hospitality.
} 


\section{Introduction}

The provision of goods and services requires tasks to be accomplished by the factors of production. In other words, what matters in the process of production is not the mere presence of factors but what they do. The present paper accounts for this observation and develops a novel interpretation of the neoclassical production sector where capital and labor perform factor-specific tasks to produce output. A task may be thought of as a unit of work activity that contributes to the production of output.

The task-based interpretation of the neoclassical production sector sheds new light on the intricate relationship between factor-augmenting technical change and relative and absolute factor prices. In particular, we establish that the effect of factor-augmenting technical change on relative and absolute factor prices can be decomposed into a productivity effect and a market size effect of opposite sign. These effects appear since the task-based approach distinguishes between the demands for tasks and the demands for factors. This perspective provides a new intuition for the emergence of relative and absolute factor biases and the role of the elasticity of substitution. The findings of the present paper are derived in the following steps.

Section 2 introduces tasks into the neoclassical production sector and defines the competitive equilibrium. The novel features include a production function defined over factor specific tasks and input coefficients that provide the link between each task and the required amount of the factor that accomplishes it. Taking tasks as the primitive means that firms maximize profits to find the optimal number of tasks performed by capital and labor. These choices determine their factor demands as well as their output supplies.

Section 3 analyzes the model under the assumption that factor specific tasks are homogeneous in the sense that the productivity of factors in the performance of their respective task is the same. ${ }^{1}$ We establish the existence of a unique competitive equilibrium in Section 3.1 and show that the resulting allocation coincides with the one obtained for a neoclassical production sector where firms' production technology is directly defined over their efficient capital and labor inputs. In this sense the suggested interpretation based on tasks provides an intellectual underpinning of the neoclassical production sector used in the literature.

Section 3.2 develops a new intuition for the way in which factor-augmenting technical change affects relative and absolute equilibrium factor prices. In fact, our main finding

\footnotetext{
${ }^{1}$ As will become clear in Section 2, the analytical framework developed here accounts for both homogeneous and heterogeneous tasks. However, to get as close as possible to the canonical setup of the neoclassical production sector we focus on homogeneous tasks in the main text. The supplementary material, relegated to an additional appendix (Section 6), has a detailed analysis of a case with heterogenous tasks.
} 
is that the relative factor bias of technical change hinges on the tension between a positive productivity effect and a negative market size effect. According to the former, the relative price of a factor increases if its productivity increases. According to the latter, a higher relative productivity of a factor increases the relative demand for tasks provided by this factor since the supply of this factor in efficiency units increases. Accordingly, the equilibrium in the factor markets requires the equilibrium task-intensity, i.e., the ratio of the number of tasks provided by each factor, to adjust. At the level of firms the market size effect requires a substitution of tasks and, quite intuitively, the degree of substitution is determined by the elasticity of substitution. This leads to the conclusion for the CES production function, that the productivity effect dominates the market size effect if and only if the elasticity of substitution exceeds unity.

This paper builds on and contributes to at least two branches of the literature interested in the possible implications of technical change in factor markets. The first branch takes a new look at the old question of factor-price biases of technical change (see, e.g., Acemoglu (2007), Acemoglu (2010)). While technical change is exogenous in the present paper, the results we derive are informative on the conditions under which technical change increases or decreases the relative and the absolute marginal product of factors. As to relative marginal products, our emphasis on tasks provides an intuitive interpretation of the role played by the elasticity of substitution for the sign of these effects in terms of a productivity and a market size effect.

Second, we contribute to the literature that studies relevant economic phenomena emphasizing the role of tasks. Examples include Dornbusch, Fischer, and Samuelson (1980) with an application to international trade patterns, Champernowne (1961) and Zeira (1998) with a focus on economic growth and capital-labor substitution, or Acemoglu and Autor (2011) who study employment and earnings profiles across skill groups. Related is also the recent "endogenous growth" literature emphasizing tasks in the context of automation incentives and economic growth (see, e. g., Irmen (2017), Irmen and Tabakovic (2017), Irmen (2018), or Acemoglu and Restrepo (2018)). Here, we show that the neoclassical production sector has a simple interpretation in terms of tasks that provides new intuitions and new predictions.

The remainder of this paper is organized as follows. Sections 2 introduces tasks into the neoclassical production sector. Section 3 derives our main results for homogeneous tasks. Section 4 concludes. Proofs are relegated to Section 5, the appendix.

\section{Tasks in the Neoclassical Production Sector}

Consider a static economy endowed with capital, $K \in \mathbb{R}_{++}$and labor, $L \in \mathbb{R}_{++}$. There is one final good produced by a competitive production sector with a continuum of identical firms. We represent this sector by the unit interval and use $i$ to index firms, i. e., $i \in[0,1]$. The final good serves as numéraire. 
All firms have access to the same production technology. On the one hand, the technology describes the relationship between the accomplishment of tasks and the resulting output. On the other hand, it specifies the input requirements of tasks.

There are two distinct types of tasks, one is performed by capital, the other by labor. ${ }^{2}$ Let $m \in \mathbb{R}_{+}$denote tasks performed by capital and $n \in \mathbb{R}_{+}$tasks performed by labor. All firms have access to the same neoclassical production function $F: \mathbb{R}_{+}^{2} \rightarrow \mathbb{R}_{+}$, i.e., output of firm $i, Y(i)$, is

$$
Y(i)=F(M(i), N(i))
$$

where $M(i)$ and $N(i)$ denote the respective total amount of tasks performed by capital and labor in firm $i$. In other words, if firm $i$ performs all tasks $m \in[0, M(i)]$ and $n \in$ $[0, N(i)]$ then the maximum output it produces is $F(M(i), N(i))$. Since $F(M(i), N(i))$ is a neoclassical production function, it exhibits constant returns to scale and positive, yet decreasing marginal products to both types of tasks. ${ }^{3}$

Task $m$ requires $k(m)=1 / b(m)>0$ units of capital. Similarly, task $n$ requires $l(n)=1 / a(n)>0$ units of labor. Hence, $b(m)>0$ is the productivity of capital in the performance of task $m$ whereas $a(n)>0$ is the productivity of labor in the performance of task $n$.

Competitive firms take factor prices, i.e., the real rental rate of capital, $R$, and the real wage, $w$, as given and maximize profits. Hence, they choose the plan

$$
\left(M(i), N(i),[k(m)]_{m \in[0, M(i)]},[l(n)]_{n \in[0, N(i)]}\right)
$$

comprising the total number of tasks performed by either capital or labor and the amount of capital or labor to accomplish each task. These choices are to maximize profits

$$
\pi(i)=F(M(i), N(i))-C(i)
$$

where

$$
C(i)=R \int_{0}^{M(i)} k(m) d m+w \int_{0}^{N(i)} l(n) d n
$$

is firm $i$ 's total factor costs comprising the costs of capital and labor incurred to perform $M(i)$, respectively, $N(i)$, tasks.

\footnotetext{
${ }^{2}$ The labels "capital" and "labor" are just for convenience. Mathematically, these two factors may represent any pair of distinguishable inputs. The analytical setup developed here extends readily to more than two inputs each with a corresponding task type.

${ }^{3}$ To include the CES production function, I dispense with Inada conditions. However, throughout I assume that the $\lim _{M(i) \rightarrow 0} \partial F(M(i), N(i)) / \partial M(i)$ and $\lim _{N(i) \rightarrow 0} \partial F(M(i), N(i)) / \partial N(i)$ are sufficiently high to deliver an interior solution to the profit-maximization problem of firms.
} 
Let

$$
K(i)=\int_{0}^{M(i)} k(m) d m \text { and } L(i)=\int_{0}^{N(i)} l(n) d n
$$

denote firm $i$ 's the demand for capital and labor.

Factor endowments are inelastically supplied, i.e., capital owners and workers supply the services of their factors to the firms that offer the highest remuneration. Therefore, all firms must pay the same factor prices to attract inputs. Moreover, capital and labor are perfectly divisible across tasks of the respective type. Then, the factor market clearing conditions are

$$
\begin{gathered}
R \geq 0, \quad \int_{0}^{1} K(i) d i \leq K, \quad \text { and } \quad R\left(\int_{0}^{1} K(i) d i-K\right)=0, \\
w \geq 0, \quad \int_{0}^{1} L(i) d i \leq L \quad \text { and } \quad w\left(\int_{0}^{1} L(i) d i-L\right)=0 .
\end{gathered}
$$

They account for the fact that an excess supply is only possible if the respective factor price is zero. The competitive equilibrium is then defined as follows.

\section{Definition 1 (Competitive Equilibrium)}

The competitive equilibrium is a set of plans (2.2) for all firms $i \in[0,1]$ and factor prices $\left(R^{*}, w^{*}\right) \geq 0$ such that all plans maximize profits (2.3) for given factor prices, and the factor market clearing conditions (2.6) hold.

\section{Homogeneous Tasks}

Let tasks be homogenous in the sense that the productivity of capital and labor in the performance of their respective tasks is independent of $m$ and $n$.

Assumption 1 (Homogeneous Tasks) $b(m)=b>0$ and $a(n)=a>0$.

\subsection{The Competitive Equilibrium}

With Assumption 1, firm i's factor costs (2.4) are

$$
C(i)=\frac{R M(i)}{b}+\frac{w N(i)}{a} \text {. }
$$


Using the latter in (2.3) leads to the first-order (sufficient) conditions for an interior solution

$$
\begin{aligned}
\frac{\partial \pi(i)}{\partial M(i)} & =\frac{\partial F(M(i), N(i))}{\partial M(i)}-\frac{R}{b}=0, \\
\frac{\partial \pi(i)}{\partial N(i)} & =\frac{\partial F(M(i), N(i))}{\partial N(i)}-\frac{w}{a}=0
\end{aligned}
$$

for all $i \in[0,1]$. Hence, the marginal value product of task $M(i)$ is equal to its marginal cost. Since performing a task with capital requires $1 / b$ units of capital, the latter is equal to $R / b$. A similar interpretation applies to labor.

\section{Proposition 1 (Competitive Equilibrium with Homogeneous Tasks)}

The competitive equilibrium involves aggregate quantities of tasks $M^{*}=b K$ and $N^{*}=a L$, strictly positive factor prices $R^{*}=\partial F\left(M^{*}, N^{*}\right) / \partial M(i)>0, w^{*}=\partial F\left(M^{*}, N^{*}\right) / \partial N(i)>0$, and aggregate output $F\left(M^{*}, N^{*}\right)=F(b K, a L)$. The equilibrium task intensity of all firms is equal to $(M(i) / N(i))^{*}=M^{*} / N^{*}=b K /(a L)$. The scale of each firm remains indeterminate.

Proposition 1 shows that the economy's efficient amount of capital and labor, i. e., $b K$ and $a L$, coincides with the respective equilibrium amount of tasks performed by capital and labor. Hence, in equilibrium, the respective task productivity $b$ and $a$ is factoraugmenting. Moreover, the equilibrium task-intensity $(M(i) / N(i))^{*}$ of all firms is equal to the economy's efficient capital intensity $b K /(a L)$. Since firms operate under constant returns to scale, their scale of production remains indeterminate. Figure 3.1 provides an illustration of the equilibrium configuration.

Two remarks are in order. First, observe that the competitive equilibrium of Proposition 1 delivers the same allocation as would be obtained if firms had direct access to the production function $F(b K(i), a L(i))$, with $K(i)$ and $L(i)$ denoting firm $i$ 's demand for capital and labor. This follows since the factor-market clearing condition (2.6) applies, and profits are $\pi(i)=F(b K(i), a L(i))-R K(i)-w L(i)$. Accordingly, firm $i$ 's first-order conditions read

$$
\begin{aligned}
& \frac{\partial \pi(i)}{\partial K(i)}=\frac{\partial F(b K(i), a L(i))}{\partial K(i)}-R=0, \\
& \frac{\partial \pi(i)}{\partial L(i)}=\frac{\partial F(b K(i), a L(i))}{\partial L(i)}-w=0 .
\end{aligned}
$$

These conditions coincide with those of (3.2) since

$$
\begin{aligned}
& \frac{\partial F(b K(i), a L(i))}{\partial K(i)}=b F_{1}(b K(i), a L(i))=\left.b \cdot \frac{\partial F(M(i), N(i))}{\partial M(i)}\right|_{M(i)=b K(i), N(i)=a L(i)}, \\
& \frac{\partial F(b K(i), a L(i))}{\partial L(i)}=b F_{2}(b K(i), a L(i))=\left.b \cdot \frac{\partial F(M(i), N(i))}{\partial N(i)}\right|_{M(i)=b K(i), N(i)=a L(i)} .
\end{aligned}
$$


Figure 3.1: The Competitive Equilibrium in $(N, M)$-Space. In equilibrium, all firms produce with the same task intensity $(M(i) / N(i))^{*}=M^{*} / N^{*}$. However, $M^{*}(i)$ and $N^{*}(i)$ remain indeterminate. Possible scales for two firms $i$ and $j$ are shown. For the production sector as a whole, production occurs at minimum costs, $C^{*}$. The corresponding isocost curve is tangential to the isoquant for the output level $Y=F\left(M^{*}, N^{*}\right)$.

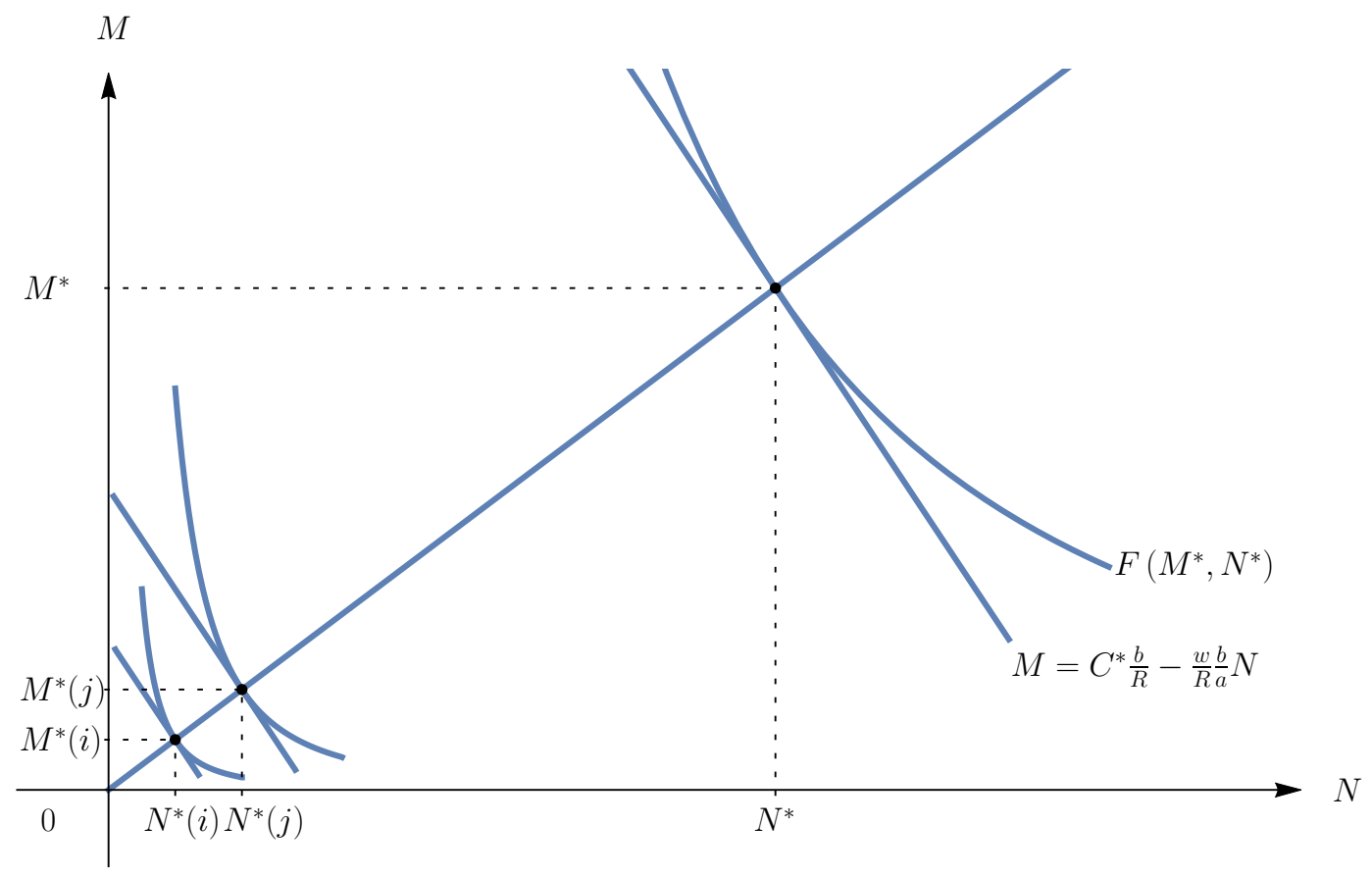

Finally, notice that the allocation given in Proposition 1 also obtains if we use the representative-firm shortcut where a single competitive firm, equipped with a production function $F(M, N)$ chooses the plan $\left(M, N,[k(m)]_{m \in[0, M]},[l(n)]_{n \in[0, N]}\right)$ in conjunction with the factor-market clearing conditions $M / b-K=0$ and $M / a-L=0$. In what follows, we shall apply this shortcut. ${ }^{4}$

\subsection{Tasks, Technology, and Factor Prices}

This section draws on Proposition 1 and shows that the equilibrium effect of technological change on relative factor prices as well as on absolute factor prices reflects the tension between a productivity effect and a market size effect of opposite sign. This finding

${ }^{4}$ Little would change in Proposition 1 and its proof if we replaced the production function of (2.1) by any homothetic function $\tilde{F}: \mathbb{R}_{+}^{2} \rightarrow \mathbb{R}_{+}$where $\tilde{F}(M(i), N(i))=h(F(M(i), N(i)))$ with $h^{\prime}(\cdot)>0>h^{\prime \prime}(\cdot)$. In fact, the equilibrium allocation and equilibrium factor price are simply obtained by replacing $F$ by $\tilde{F}$. 
appears in the task-based interpretation of the neoclassical production sector expounded in the previous sections because it distinguishes between the demands for tasks and the demands for factors. The market size effect leads to a substitution of tasks. It will therefore depend on the elasticity of substitution whereas the productivity effect does not. To highlight this distinction, let us consider a competitive economy with a representative firm equipped with a CES production function

$$
F(M, N)=\left[\gamma_{M} M^{\frac{\sigma-1}{\sigma}}+\gamma_{N} N^{\frac{\sigma-1}{\sigma}}\right]^{\frac{\sigma}{\sigma-1}},
$$

where $\sigma \in(0, \infty]$ is the elasticity of substitution between tasks and $0<\gamma_{M}, \gamma_{N}<1$ are distribution parameters satisfying $\gamma_{M}+\gamma_{N}=1$.

\subsubsection{Relative Factor Prices}

Profit-maximization with respect to $M$ and $N$ leads to the following condition on the relative price of capital

$$
\frac{R}{w}=\gamma\left(\frac{b}{a}\right)\left(\frac{M}{N}\right)^{\frac{-1}{\sigma}}
$$

or, equivalently,

$$
\ln \left(\frac{R}{w}\right)=\ln \gamma+\ln \left(\frac{b}{a}\right)-\frac{1}{\sigma} \ln \left(\frac{M}{N}\right),
$$

where $\gamma \equiv \gamma_{M} / \gamma_{N}$. Both expressions reflect the substitution effect that changing factor prices exert on the relative task intensity chosen by the representative firm. We interpret (3.6) as the (log-linear) inverse of the relative demand for tasks performed by capital. The elasticity of substitution determines the responsiveness of the relative price of capital to changes in $M / N$, i.e.,

$$
\frac{\partial \ln (R / w)}{\partial \ln (M / N)}=-\frac{1}{\sigma}
$$

Intuitively, the substitution of tasks depends on the varying conditions the firm faces in the factor markets and on its production function. The former is parameterized by the change in $R / w$, the latter by $\sigma$. A given percentage decline in $R / w$, induces a large percentage increase in $M / N$ if the substitution is technically easy to accomplish, i. e., when $\sigma$ is large.

Next, consider the equilibrium relative demand for tasks performed by capital. According to Proposition 1 it coincides with the equilibrium task intensity equal to $M^{*} / N^{*}=$ $b K /(a L)$, or equivalently

$$
\ln \left(\frac{M^{*}}{N^{*}}\right)=\ln \left(\frac{b}{a}\right)+\ln \left(\frac{K}{L}\right) .
$$

Hence, in light of (3.6) and (3.8) the following result concerning the effect of a change in the relative productivity of capital on the equilibrium relative price of capital follows. 


\section{Proposition 2 (Tasks, Technology, and Relative Factor Prices)}

Consider the competitive equilibrium of Proposition 1. Then, it holds that

$$
\frac{d \ln \left(R^{*} / w^{*}\right)}{d \ln (b / a)}=\underbrace{\frac{\partial \ln \left(R^{*} / w^{*}\right)}{\partial \ln (b / a)}}_{\text {Productivity Effect }}+\underbrace{\frac{\partial \ln \left(R^{*} / w^{*}\right)}{\partial \ln (M / N)} \cdot \frac{\partial \ln \left(M^{*} / N^{*}\right)}{\partial \ln (b / a)}}_{\text {Market Size Effect }} .
$$

According to Proposition 2 the total effect of changing the relative productivity of capital, $b / a$, on equilibrium relative factor prices, $R^{*} / w^{*}$, can be decomposed into a productivity effect and a market size effect. This is illustrated in Figure 3.2.

From (3.6) the productivity effect is strictly positive as

$$
\frac{\partial \ln \left(R^{*} / w^{*}\right)}{\partial \ln (b / a)}=1
$$

Given $M^{*} / N^{*}$, the productivity effect captures that an increase in $b / a$ leads to a proportionate increase in the relative marginal product of tasks performed by capital. Accordingly, an increase in $R^{*} / w^{*}$ is called for so that profit-maximizing firms desire to perform $M^{*} / N^{*}$ tasks. ${ }^{5}$

The second summand on the right-hand side of (3.9) is the market size effect. It is a general equilibrium effect and the product of two factors. The first factor reflects the change in relative efficient factor supplies. According to (3.8), we have $\partial \ln \left(M^{*} / N^{*}\right) / \partial \ln (b / a)=$ 1 , i. e., changing the (relative) market size of efficient capital leads to a proportionate increase in the relative equilibrium demand for tasks performed by capital to maintain full employment. The second factor captures the induced substitution of tasks and its compatibility with profit-maximizing behavior. In accordance with (3.7) the elasticity of substitution determines the extent to which factor prices have to adjust. Hence, the market size effect is equal to

$$
\frac{\partial \ln (R / w)}{\partial \ln (M / N)} \cdot \frac{\partial \ln \left(M^{*} / N^{*}\right)}{\partial \ln (b / a)}=-\frac{1}{\sigma}
$$

\footnotetext{
5Intuitively, the productivity effect leaves the position of the isocost curve shown in Figure 3.1 unchanged. Indeed, the cost function associated with the CES production function of (3.4) is

$$
C^{*}=\left[\gamma_{M}^{\sigma}\left(\frac{b}{R}\right)^{\sigma-1}+\gamma_{N}^{\sigma}\left(\frac{a}{w}\right)^{\sigma-1}\right]^{\frac{1}{1-\sigma}}
$$

This implies an isocost curve

$$
M=\left[\gamma_{M}^{\sigma}+\gamma_{N}^{\sigma}\left(\frac{b w}{a R}\right)^{1-\sigma}\right]^{\frac{1}{1-\sigma}}-\left(\frac{b w}{a R}\right) N .
$$

Hence, adjustments that satisfy (3.10) leave $b w /(a R)$ unchanged.
} 
Figure 3.2: Relative Factor Prices and the Increase in $b / a$ - the Case of $\sigma>1$. The production sector starts at point $E$ with $b / a$ and moves to point $E^{\prime}$ with $(b / a)^{\prime}$ where $(b / a)^{\prime}>b / a$. Due to the productivity effect the relative demand for tasks shifts upwards from $E$ to $P$. The general equilibrium effect implies a downward move along the relative demand from $E$ to $Q$. The total effect is the sum of these effects, i. e., the move from $E$ to $E^{\prime}$. Since $\sigma>1$ we obtain $\left(R^{*} / w^{*}\right)^{\prime}>R^{*} / w^{*}$.

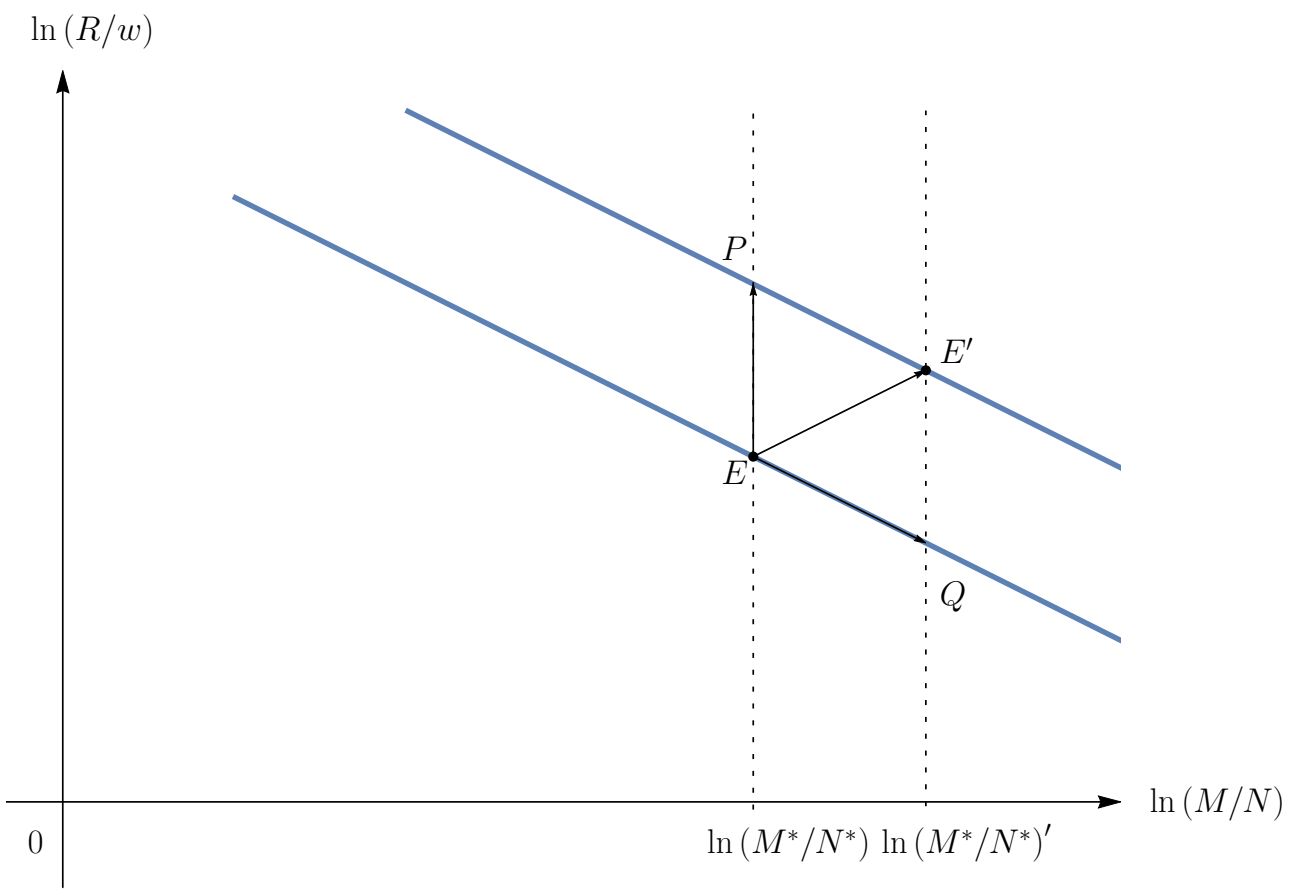

In light of (3.10) and (3.11) the total effect is then equal to

$$
\frac{d \ln (R / w)}{d \ln (b / a)}=1-\frac{1}{\sigma}
$$

and reflects the tension between the positive productivity and the negative market size effect. While the former leads to a proportionate increase in the relative price of capital, the latter implies a decline in the relative price of capital which may be more or less than proportionate depending on whether $\sigma<1$ or $\sigma>1$. The particularity of the Cobb-Douglas production function is that $\sigma=1$. In this case, the market size effect implies a proportionate decline in the relative price of capital such that the total effect of the relative productivity change on relative factor prices is zero.

To sum up, the task-based interpretation of the neoclassical production sector proposed here provides an explicit intuition for why an increase in $b / a$ is biased towards labor if $\sigma<1$ and towards capital if $\sigma>1$, i. e.,

$$
\frac{d\left(R^{*} / w^{*}\right)}{d(b / a)} \gtreqless 0 \quad \Leftrightarrow \quad \sigma \gtreqless 1 .
$$


The reason is that $\sigma$ determines the strength of the response of $R^{*} / w^{*}$ to the necessary substitution of tasks induced by a change in the relative supply of efficient capital. This response may or may not dominate the productivity effect of a change in $b / a .^{6}$

It is worth contrasting this intuition with the one proposed in the canonical neoclassical production sector where a representative competitive firm has access to the CES defined over factors in efficiency units (see, e. g., Acemoglu (2009), Chapter 15). If

$$
F(b K, a L)=\left[\gamma_{M}(b K)^{\frac{\sigma-1}{\sigma}}+\gamma_{N}(a L)^{\frac{\sigma-1}{\sigma}}\right]^{\frac{\sigma}{\sigma-1}}
$$

then in equilibrium the equivalent of (3.5) is $R^{*} / w^{*}=\gamma(b / a)^{(\sigma-1) / \sigma}(K / L)^{-1 / \sigma}$. Here, the intuition goes that relative capital-augmenting technical change is biased towards labor if $\sigma<1$ since an increase of $b / a$ implies a decline in the relative demand of labor. In other words, a fixed amount of capital and labor will find employment only if $R / w$ falls. However, this intuition remains elusive on the economics of why $\sigma$ determines the sign of $d(R / w) / d(b / a)$. The task-based interpretation introduced in the present paper fills this gap.

\subsubsection{Absolute Factor Prices}

Next, consider the effect of factor productivity on the absolute level of equilibrium factor prices. ${ }^{7}$ Here, we confine attention to the rental rate of capital. A symmetric argument applies to the equilibrium real wage. From (3.2) and factor market clearing, the equilibrium rental rate of capital satisfies

$$
R^{*}=b \cdot \frac{\partial F\left(M^{*}, N^{*}\right)}{\partial M}, \quad \text { where } M^{*}=b K \text { and } N^{*}=a L
$$

Hence, the effect of changing the productivity of capital or the one of labor on $R^{*}$ is

$$
\begin{aligned}
& \frac{d R^{*}}{d b}=\frac{\partial F\left(M^{*}, N^{*}\right)}{\partial M}+b \cdot \frac{\partial^{2} F\left(M^{*}, N^{*}\right)}{\partial M^{2}} \frac{\partial M^{*}}{\partial b} \gtreqless 0, \\
& \frac{d R^{*}}{d a}=b \cdot \frac{\partial^{2} F\left(M^{*}, N^{*}\right)}{\partial M \partial N} \cdot \frac{\partial N^{*}}{\partial a}>0 .
\end{aligned}
$$

The first line suggests that the effect of $b$ on $R^{*}$ can be decomposed into two effects of opposite sign. The first is a positive productivity effect. As $b$ increases, the effect on $R^{*}$ is equal to the marginal product of task $M^{*}$, which is positive. The second is a

\footnotetext{
${ }^{6}$ All results of Section 3.2.1 go through if we allow for decreasing returns to scale and replace the CES of $(3.4)$ by $\tilde{F}(M, N)=[F(M, N)]^{v}$, where $0<v<1$. Since $\tilde{F}(M, N)$ is homothetic, Proposition 1 continues to hold (see, Footnote 4 ).

7See Irmen (2014) for an analysis of the effect of factor-augmenting technical change on factor prices in the canonical neoclassical production sector.
} 
market size effect. As $b$ increases, more tasks must be performed by capital to maintain full employment. Since these tasks are associated with a lower marginal product, the market size effect is negative. For the CES of (3.4) it is straightforward to show that the market size effect may dominate the productivity effect for values of the elasticity of substitution that are sufficiently smaller than one. More precisely, it holds that

$$
\frac{d R^{*}}{d b} \gtreqless 0 \Leftrightarrow \sigma \gtreqless \frac{1}{\gamma\left(\frac{M^{*}}{N^{*}}\right)^{\frac{\sigma-1}{\sigma}}+1} .
$$

For instance, if $\gamma_{M}=\gamma_{N}=\sigma=1 / 2$ we have $d R^{*} / d b<0$ for $M^{*} / N^{*}>1$.

The second line of (3.15) shows that the effect of $a$ on $R^{*}$ works only through a positive market size effect. A higher productivity of labor means that $N^{*}$ must increase to maintain full employment of labor. Then, the complementarity of both tasks in the neoclassical production function implies that $R^{*}$ unequivocally increases. ${ }^{8}$

\section{Concluding Remarks}

This paper introduces tasks in a straightforward way into the neoclassical production sector. Section 2 develops the general framework. Its application leads to novel insights on the intricate relationship between technical progress and (relative and absolute) factor prices. The focus on tasks suggests that factor-augmenting technical progress gives rise to a tension between a positive productivity effect and a negative market size effect. In common parlance, the former is a direct effect, the latter a general equilibrium effect.

If tasks are homogeneous then profit-maximizing behavior delivers a log-liner (inverse) relative demand for tasks, and factor markets imply a log-linear relationship between the equilibrium relative demand for tasks and relative efficient factor supplies. Then, the productivity effect is proportionate whereas the elasticity of substitution determines whether the market size effect is more or less than proportionate. The tension of a productivity and a market size effect also drives the effect of technical progress on absolute factor prices. Here, an increase in the productivity of one factor may reduce the factor price of this factor if the elasticity of substitution is sufficiently smaller than unity.

\footnotetext{
${ }^{8}$ Complementarity in the sense that $\partial^{2} F(M, N) / \partial M \partial N>0$ follows directly from the defining properties of the neoclassical production function. With slight modifications, the results of Section 3.2.2 go through under decreasing returns to scale as suggested in Footnote 6. As to the sign of $d R^{*} / d b$, we have to replace $\gamma$ on the right hand side of (3.16) by $\tilde{\gamma} \equiv v \gamma$. The sign of $d R^{*} / d a$ in (3.15) is preserved as long as $\sigma<1 /(1-v)$.
} 


\section{Appendix: Proofs}

\subsection{Proof of Proposition 1}

Consider firm $i$ 's demand for capital. With (2.5), we have $K(i)=M(i) / b$. Hence, for $R>0$, (2.6) delivers $\int_{0}^{1} M(i) d i=b K=M^{*}$. Similarly, one derives for labor and $w>0$ that $\int_{0}^{1} N(i) d i=a L=N^{*}$. Next consider the first-order conditions (3.2). Since the derivatives $\partial F(M(i), N(i)) / \partial M(i)$ and $\partial F(M(i), N(i)) / \partial N(i)$ are homogeneous of degree zero, these conditions imply $M(i) / N(i)=M\left(i^{\prime}\right) / N\left(i^{\prime}\right)$ for all $i, i^{\prime} \in[0,1]$ and $i \neq i^{\prime}$. Therefore, it holds that $M(i) / N(i)=\left[M(i)+M\left(i^{\prime}\right)\right] /\left[N(i)+N\left(i^{\prime}\right)\right]$ and

$$
\left(\frac{M(i)}{N(i)}\right)^{*}=\frac{\int_{0}^{1} M(i) d i}{\int_{0}^{1} N(i) d i}=\frac{b K}{a L}=\frac{M^{*}}{N^{*}},
$$

where the third equality uses the factor market clearing condition (2.6). Accordingly, the equilibrium task intensity, aggregate equilibrium output, and equilibrium factor prices are as stated in Proposition 1. Since $M^{*}$ and $N^{*}$ are positive and finite, we have $R^{*}>0$ and $w^{*}>0$. Since $\pi(i)$ is homogenous of degree one in $(M(i), N(i))$, the absolute levels $M^{*}(i)$ and $N(i)^{*}$ remain indeterminate.

\subsection{Proof of Proposition 2}

In equilibrium (3.6) becomes

$$
\ln \left(\frac{R^{*}}{w^{*}}\right)=\ln \gamma+\ln \left(\frac{b}{a}\right)-\frac{1}{\sigma} \ln \left(\frac{M^{*}}{N^{*}}\right) .
$$

In light of (3.8) the proposition follows. 


\section{References}

Acemoglu, D. (2007): “Equilibrium Bias of Technology," Econometrica, 75(5), 1371-1410. Princeton, New Jersey.

(2010): “When Does Labor Scarcity Encourage Innovation?," Journal of Political Economy, 118(6), 1037-1078.

Acemoglu, D., and D. Autor (2011): “Skills, Tasks and Technologies: Implications for Employment and Earnings," in Handbook of Labor Economics - Volume 4, ed. by O. Ashenfelter, and D. Card, p. 1043.

Acemoglu, D., And P. Restrepo (2018): “The Race between Man and Machine: Implications of Technology for Growth, Factor Shares, and Employment," American Economic Review, 108(6), 1488-1542.

Champernowne, D. G. (1961): "A Dynamic Growth Model Involving a Production Function," in The Theory of Capital - Proceedings of a Conference Held by the International Economic Association, ed. by F. A. Lutz, and D. C. Hague, pp. 223-244. MacMillian (Reprinted 1963), London.

De La Grandville, O. (2017): Economic Growth - A Unified Approach. Cambridge University Press, Cambridge, New York.

Dornbusch, R., S. Fischer, and P. A. Samuelson (1980): “Heckscher- Ohlin Trade Theory with a Continuum of Goods," The Quarterly Journal of Economics, 95(2), 203-24.

Irmen, A. (2014): "Real Factor Prices and Factor-Augmenting Technical Change," The B.E. Journal of Macroeconomics, 14(1), 1-27.

- (2017): "Capital- and Labor-Saving Technical Change in an Aging Economy," International Economic Review, 58(1), 261-285.

(2018): "Automation, Leisure, and Growth in the Era of Population Aging," draft, CREA, University of Luxembourg.

Irmen, A., And A. TABaKovic (2017): "Endogenous Capital- and Labor-Augmenting Technical Change in the Neoclassical Growth Model," Journal of Economic Theory, 170, $346-384$.

ZEIRA, J. (1998): “Workers, Machines, And Economic Growth," Quarterly Journal of Economics, 113(4), 1091-1117. 


\section{Appendix: Supplementary Results - Heterogeneous Tasks}

Let us relax Assumption 1 and allow for tasks that are heterogeneous with respect to their input requirements.

Assumption 2 (Heterogeneous Tasks) $b(m)=b e^{-\delta m}$ and $a(n)=a e^{-\delta n}$, where $\delta>0$.

Assumption 2 has at least three important economic implications. First, tasks are ordered so that low values of $m$ and $n$ correspond to low input requirements, i. e., $k(m)=e^{\delta m} / b$ with $k^{\prime}(m)>0$ for $m \geq 0$ and $l(n)=e^{\delta n} / a$ with $l^{\prime}(n)>0$ for $n \geq 0$. One may think of tasks becoming more and more complex as $m$ or $n$ increases. Therefore, input requirements increase. Second, the parameter $\delta$ determines how fast these input requirements increase in $m$ or $n$. Hence, $\delta$ is a measure of the degree of heterogeneity in terms of diminishing returns including the case of homogeneous tasks with $b(m)=b$ and $a(n)=a$ for $\delta=0$. Third, increasing $b$ or $a$ means a higher productivity of the respective factor across all tasks. Hence, $b$ or $a$ are factor-augmenting with the caveat that their productivity enhancing effect is higher for tasks in which the respective factor is already more productive, i. e., $\partial^{2} b(m) / \partial b \partial m<0$ and $\partial^{2} a(n) / \partial a \partial n<0$.

\subsection{The Competitive Equilibrium}

Firm i's factor costs (2.4) are now equal to

$$
C(i)=\frac{R}{b} \int_{0}^{M(i)} e^{\delta m} d m+\frac{w}{a} \int_{0}^{N(i)} e^{\delta n} d n
$$

Using the latter in (2.3) leads to the first-order (sufficient) conditions for an interior solution

$$
\begin{aligned}
\frac{\partial \pi(i)}{\partial M(i)} & =\frac{\partial F(M(i), N(i))}{\partial M(i)}-\frac{R}{b} e^{\delta M(i)}=0, \\
\frac{\partial \pi(i)}{\partial N(i)} & =\frac{\partial F(M(i), N(i))}{\partial N(i)}-\frac{w}{a} e^{\delta N(i)}=0,
\end{aligned}
$$

for all $i \in[0,1]$ which extends (3.2) to the case of $\delta>0$. Intuitively, the cost of the respective marginal task increases in $M(i)$ and $N(i)$ reflecting higher input requirements for the marginal than for inframarginal tasks. In view of Definition 1 the following proposition obtains.

Proposition 3 (Competitive Equilibrium with Heterogeneous Tasks)

There is a unique competitive equilibrium involving aggregate quantities of tasks $M^{*}=\ln (1+\delta b K) / \delta$ and $N^{*}=\ln (1+\delta a L) / \delta$, strictly positive factor prices $R^{*}=b\left(\partial F\left(M^{*}, N^{*}\right) / \partial M\right) / e^{\delta M^{*}}>0, w^{*}=a\left(\partial F\left(M^{*}, N^{*}\right) / \partial N\right) / e^{\delta N^{*}}>0$, and aggregate output equal to $F(\ln (1+\delta b K), \ln (1+\delta a L)) / \delta$. All firms produce at the same scale, i.e., $M^{*}(i)=M^{*}$ and $N^{*}(i)=N^{*}$.

\section{Proof of Proposition 3}

We start proving that $M(i)=M(j)=M^{*}$ and $N(i)=N(j)=N^{*}$ for all $i, j \in[0,1]$ and $i \neq j$ holds in equilibrium. Since $C(i)=C(M(i), N(i))$ is strictly convex, profits $\pi(i)=\pi(M(i), N(i))$ are strictly concave. Assume to the contrary that two firms $i$ and $j$ choose $(M(i), N(i)) \neq(M(j), N(j))$. This is only possible if $\pi(M(i), N(i))=\pi(M(j), N(j))$. Now, consider $(M, N)=\mu(M(i), N(i))+(1-\mu)(M(j), N(j))$ for some $\mu \in(0,1)$. Since profits are strictly concave we have $\pi(M, N)>\mu \pi(M(i), N(i))+(1-\mu) \pi(M(j), N(j))$. Therefore, $\pi(M, N)>\pi(M(i), N(i))=\pi(M(j), N(j))$ which is a contradiction. Hence, for all $i \in[0,1]$ we must have $(M(i), N(i))=(M, N)$.

As a consequence, the aggregate demand for capital of all firms is $\int_{0}^{M} e^{\delta m} d m / b=\left(e^{\delta M}-1\right) /(\delta b)$. Hence, for $R>0$, (2.6) delivers $M^{*}$ as indicated in the proposition. Similarly, one derives $N^{*}$. The first-order conditions (6.2) deliver $R^{*}$ and $w^{*}$ as indicated. Since $F(\cdot)$ has constant returns to scale, $F\left(M^{*}, N^{*}\right)=F(\ln (1+\delta b K), \ln (1+\delta a L)) / \delta$.

Proposition 3 exploits the fact that the marginal cost of tasks increases under Assumption 2. Therefore, firm profits become strictly concave, and the equilibrium scale of each firm can be pinned down. Since a higher $\delta$ increases the input 
requirements of both factors, we have $\partial M^{*} / \partial \delta<0$ and $\partial N^{*} / \partial \delta<0$. As a consequence, aggregate output increases as $\delta$ declines with $\lim _{\delta \rightarrow 0} F(\ln (1+\delta b K), \ln (1+\delta a L)) / \delta=F(b K, a L) .9$

The equilibrium allocation of Proposition 3 is the same as would be obtained if firms had direct access to the production function $F(\ln (+b K(i)), \ln (1+a L(i))) / \delta$, where $K(i)$ and $L(i)$ denote firm demands for capital and labor. However, here the efficient amount of capital (labor) of the economy is equal to the equilibrium amount of tasks performed by capital (labor) and no longer equal to $b K$ or $a L .{ }^{10}$

\subsection{Tasks, Technology, and Factor Prices}

This section studies the relationship between factor prices and the technology represented by the parameters $b$ and $a$. The results are then compared to those derived for homogeneous tasks in Section 3. We focus on the CES of (3.4).

\subsubsection{Relative Factor Prices}

Evaluated at the competitive equilibrium of Proposition 3, the first-order conditions (6.2) deliver the expressions for the relative price of capital in equilibrium as

$$
\begin{aligned}
\frac{R^{*}}{w^{*}} & =\gamma\left(\frac{b\left(M^{*}\right)}{a\left(N^{*}\right)}\right)\left(\frac{M^{*}}{N^{*}}\right)^{\frac{-1}{\sigma}} \\
& =\gamma\left(\frac{b}{a}\right)\left(\frac{e^{-\delta M^{*}}}{e^{-\delta N^{*}}}\right)\left(\frac{M^{*}}{N^{*}}\right)^{\frac{-1}{\sigma}}
\end{aligned}
$$

Hence, the relative price of capital is determined by the distribution parameter, $\gamma$, the relative productivity of the respective marginal tasks, and the factor reflecting the substitutability of tasks. Task heterogeneity makes the relevance of the relative factor productivity of the marginal tasks explicit. In fact, if tasks are homogeneous then $R^{*} / w^{*}$ will also depend on the relative factor productivity of the marginal tasks. However, since tasks do not differ this relative factor productivity is equal to $b / a$ as shown in (3.5). It is worth noting that the heterogeneity of tasks does not affect the firm's willingness to substitute tasks of the two types. ${ }^{11}$ It will, however, influence the effect of changes in $b$ and $a$ on the adjustment of $M^{*}$ and $N^{*}$ through the market size effect. Indeed, with Proposition 3 one readily verifies that

$$
\frac{\partial \ln M^{*}}{\partial \ln b}<1 \text { and } \frac{\partial \ln N^{*}}{\partial \ln a}<1
$$

Unlike for homogeneous tasks, the response of $M^{*}$ and $N^{*}$ to percentage changes of the respective productivity parameters is less than proportionate reflecting increasing factor requirements of the additional tasks necessary to maintain full employment.

To study the effect of $b$ and $a$ on the relative price of capital, we take logs on both sides of (6.3) and obtain

$$
\ln \left(R^{*} / w^{*}\right)=\ln \gamma+\ln (b / a)-\delta\left(M^{*}-N^{*}\right)-\frac{\ln \left(M^{*} / N^{*}\right)}{\sigma} .
$$

${ }^{9}$ Observe that $F(\ln (1+\delta b K), \ln (1+\delta a L)) / \delta$ is not homothetic in $(b K, a L)$. Therefore, the findings on technical change and factor prices below are quite different from and have different intuitions than those we state in the footnotes 4 - 8 for a CES with decreasing returns to efficient factor supplies.

${ }^{10}$ Notice that the 'number' of firms will affect aggregate output and factor prices since capital and labor exhibit diminishing returns in the accomplishment of tasks. A larger number of firms implies that each firm operates at a smaller scale. The equilibrium productivity of capital and labor will then be higher and aggregate output increases. In what follows we neglect this complication to facilitate the comparison with the results derived in Section 3 .

${ }^{11}$ On the semantic front, (6.3) challenges the interpretation of $\sigma$ as the elasticity of substitution (see, de La Grandville (2017) for a careful discussion of this concept). In the present context, $\sigma$ measures the effect of $\partial \ln \left(M^{*} / N^{*}\right)$ on $\partial \ln \left(R^{*} / w^{*}\right)$ given the relative productivity of tasks. Accounting for the fact that the relative productivity of tasks depends on the marginal tasks adds a new dimension to this relationship. We refer to the latter as a new facet of the productivity effect defined in Section 3 . 
Due to the presence of diminishing returns in the accomplishment of tasks, the inverse of the relative demand for tasks performed by capital is no longer log-linear. Nevertheless, there is a relationship between $R^{*} / w^{*}$ and the productivity levels $b$ and $a$.

Proposition 4 (Tasks, Technology, and Relative Factor Prices with Heterogeneous Tasks)

Consider the competitive equilibrium of Proposition 3. Then, it holds that

$$
\begin{aligned}
& \frac{d\left(R^{*} / w^{*}\right)}{d \ln b}=\left(1-\delta M^{*} \frac{\partial \ln M^{*}}{\partial \ln b}\right)-\frac{1}{\sigma} \frac{\partial \ln M^{*}}{\partial \ln b}, \\
& \frac{d\left(R^{*} / w^{*}\right)}{d \ln a}=\left(-1+\delta N^{*} \frac{\partial \ln N^{*}}{\partial \ln a}\right)+\frac{1}{\sigma} \frac{\partial \ln N^{*}}{\partial \ln a} .
\end{aligned}
$$

Moreover, there are critical values $\bar{\sigma}_{b}=\bar{\sigma}_{b}(\delta)>1$ with $d \bar{\sigma}_{b} / d \delta>0$ and $\bar{\sigma}_{a}=\bar{\sigma}_{a}(\delta)>1$ with $d \bar{\sigma}_{a} / d \delta>0$ such that

$$
\begin{aligned}
& \frac{d \ln (R / w)}{d \ln b} \gtreqless 0 \Leftrightarrow \sigma \gtreqless \bar{\sigma}_{b}, \\
& \frac{d \ln (R / w)}{d \ln a} \gtreqless 0 \Leftrightarrow \sigma \lesseqgtr \bar{\sigma}_{a} .
\end{aligned}
$$

\section{Proof of Proposition 4}

The statements of equation (6.6) are immediate from (6.5). Computing the respective effects delivers with $z \equiv \delta b K$

$$
\delta M^{*} \frac{\partial \ln M^{*}}{\partial \ln b}=\frac{z}{1+z} \quad \text { and } \quad \frac{1}{\sigma} \frac{\partial \ln M^{*}}{\partial \ln b}=\frac{1}{\sigma}\left(\frac{z}{1+z}\right)\left(\frac{1}{\ln (1+z)}\right) .
$$

It follows that $\bar{\sigma}_{b}=z /(\ln (1+z))>1$ which is increasing in $z$, hence, also in $\delta$. As to labor, let $x \equiv \delta a L$. Then,

$$
\delta N^{*} \frac{\partial \ln N^{*}}{\partial \ln a}=\frac{x}{1+x} \quad \text { and } \quad \frac{1}{\sigma} \frac{\partial \ln N^{*}}{\partial \ln a}=\frac{1}{\sigma}\left(\frac{x}{1+x}\right)\left(\frac{1}{\ln (1+x)}\right) .
$$

It follows that $\bar{\sigma}_{a}=x /(\ln (1+x))>1$ which is increasing in $x$, hence, also in $\delta$.

The intuition behind the first finding of Proposition 4 comes in terms of the productivity and the market size effect identified before. To see this consider the two effects of $b$ on $R^{*} / w^{*}$ as stated in (6.6). The productivity effect has now two components and is given by the expression in parentheses. The first component is a proportionate effect since $b$ increases the productivity of capital in all performed tasks. The second component captures the interaction of task productivity and the market size for tasks performed by capital. A higher $b$ increases the supply of efficient capital which requires a higher $M^{*}$ to maintain full employment. Accordingly, the productivity of capital in the marginal task declines and so does $R^{*}$. The second term in the first line of (6.6) reflects the market size effect that leads to a substitution of tasks. In line with (6.4), this effect is weaker than under homogeneous tasks since $M^{*}$ increases less than proportionately. Mutatis mutandis, this intuition also explains the effect of $a$ on $R^{*} / w^{*}$.

The results stated in (6.7) show that the simple rule (3.13) must be modified to account for task heterogeneity. ${ }^{12}$ In fact, with heterogeneous tasks, a percentage change in $b$ may decrease $R^{*} / w^{*}$ even if $\sigma>1$ since $\bar{\sigma}_{b}>1$. Moreover, the range of $\sigma$ for which this is possible increases in the degree of heterogeneity parameterized by $\delta$. This suggests that the response to changes in $\delta$ of the relative factor productivity across tasks is more pronounced than the one through the market size effect. In the same vein, a percentage increase in $a$ may increase $R^{*} / w^{*}$ even for $\sigma>1$. Finally, taking the limit $\delta \rightarrow 0$ in (6.6) delivers

$$
\left.\frac{d \ln (R / w)}{d \ln b}\right|_{\delta=0}=1-\frac{1}{\sigma} \quad \text { and }\left.\quad \frac{d \ln (R / w)}{d \ln a}\right|_{\delta=0}=-1+\frac{1}{\sigma},
$$

i. e., for $\delta>0$ but sufficiently close to zero (3.13) remains a good approximation.

\footnotetext{
${ }^{12}$ Observe that $d \ln (R / w) / d \ln (b / a)=d \ln (R / w) / d \ln b=-d \ln (R / w) / d \ln a$ since $d \ln (b / a)=d \ln b-d \ln a$. Therefore, the rule stated in (3.13) also applies to $d \ln (R / w) / d \ln b$ and with opposite sign to $d \ln (R / w) / d \ln a$.
} 
Table 1: Critical values $\hat{\sigma}_{b}$ for changing values of $\delta$.

\begin{tabular}{|c||c|c|c|c|c|c|}
\hline$\delta$ & 2 & 3 & 4 & 5 & 8 & 10 \\
\hline$\hat{\sigma}_{b}$ & 1.23 & 1.53 & 1.8 & 2.07 & 2.8 & 3.27 \\
\hline
\end{tabular}

\subsubsection{Absolute Factor Prices}

To elicit the effect of factor productivity on the absolute level of equilibrium factor prices consider the first-order conditions (6.2) and factor market clearing. The equilibrium rental rate of capital satisfies

$$
R^{*}=b\left(M^{*}\right) \cdot \frac{\partial F\left(M^{*}, N^{*}\right)}{\partial M}, \quad \text { where } M^{*}=\ln (1+\delta b K) \text { and } N^{*}=\ln (1+\delta a L) .
$$

Hence, the effect of changing $b$ or $a$ on $R^{*}$ is

$$
\begin{aligned}
\frac{d R^{*}}{d b} & =\left(\frac{\partial b\left(M^{*}\right)}{\partial b}+\frac{\partial b\left(M^{*}\right)}{\partial M} \cdot \frac{\partial M^{*}}{\partial b}\right) \cdot \frac{\partial F\left(M^{*}, N^{*}\right)}{\partial M}+b\left(M^{*}\right) \cdot \frac{\partial^{2} F\left(M^{*}, N^{*}\right)}{\partial M^{2}} \cdot \frac{\partial M^{*}}{\partial b} \gtreqless 0, \\
\frac{d R^{*}}{d a} & =b\left(M^{*}\right) \cdot \frac{\partial^{2} F\left(M^{*}, N^{*}\right)}{\partial M \partial N} \cdot \frac{\partial N^{*}}{\partial a}>0 .
\end{aligned}
$$

As for homogeneous tasks, the effect of $b$ on $R^{*}$ consist of a productivity effect and a market size effect of opposite sign. The comparison with (3.15) reveals a new channel that dampens the productivity effect: as $M^{*}$ increases in response to a higher $b$, the productivity of the marginal task declines which lowers $R^{*}$. For the CES of (3.4) this leads to

$$
\frac{d R^{*}}{d b} \gtreqless 0 \quad \Leftrightarrow \quad \sigma \gtreqless \frac{\partial \ln M^{*}}{\partial \ln b}\left(\frac{1}{\gamma\left(\frac{M^{*}}{N^{*}}\right)^{\frac{\sigma-1}{\sigma}}+1}+\delta\right),
$$

which extends (3.16) to $\delta>0$. This expression is hard to sign in general. However, since $\partial \ln M^{*} / \partial \ln b<1$ it becomes feasible that an increase in $b$ decreases $R^{*}$ even if $\sigma>1$. For instance, if $\gamma_{M}=\gamma_{N}=a L=1, b K=2$, and $\delta \geq 2$ then there is a critical value $\hat{\sigma}_{b}>1$ such that $d R^{*} / d b<0$ for all $\sigma<\hat{\sigma}_{b}$. For this set of parameters Table 1 gives some critical values $\hat{\sigma}_{b}$ for varying values of $\delta$. It suggests that a higher degree of task heterogeneity makes it easier for $d R^{*} / d b<0$ to occur even for $\sigma>1$.

Finally observe that the direction of the effect of $a$ on $R^{*}$ remains positive and driven by the complementarity of tasks in $F$. The degree of heterogeneity may affect the strength but not the direction of this effect. 